\title{
The Effect of Local Platelet Rich Plasma Therapy on the Composition of Bacterial Flora in Chronic Venous Leg Ulcer
}

\author{
WITOLD WOŹNIAK ${ }^{1 *}$, MONIKA TARNAS², TOMASZ MIŁEK ${ }^{1}$, R. KRZYSZTOF MLOSEK ${ }^{3}$ \\ and PIOTR CIOSTEK ${ }^{1}$

\begin{abstract}
${ }^{1}$ First Department of General and Vascular Surgery, Second Faculty of Medicine with the English Division and the Physiotherapy Division, Medical University of Warsaw, Warsaw, Poland

${ }^{2}$ Microbiology Department, Mazovian Bródnowski Hospital in Warsaw, Warsaw, Poland

${ }^{3}$ Department of Ultrasonographic Diagnostics and Mammography, Second Faculty of Medicine with the English Division and the Physiotherapy Division, Medical University of Warsaw, Warsaw, Poland
\end{abstract}

Submitted 22 January 2016, accepted 6 June 2016

Abstract

Microbial colonisation of chronic venous ulcers and synergism between bacterial species slow down the healing process. The study aimed at performing qualitative analysis of microbial flora in venous leg ulcers treated with platelet rich plasma (PRP). Twenty two women and twelve men aged 47-90 years were treated with PRP at our department between 2012 and 2015. Ulcer cultures collected before and after PRP therapy yielded 83 and 110 microbial isolates, respectively, of Gram positive, Gram negative bacteria and candida. Pseudomonas aueruginosa and Staphylococcus aureus were the most common pre- and post-treatment isolates. PRP therapy and increased the variety of microbial flora.

Ke y w ord s: antibacterial activity, microflora of venous leg ulcer, platelet-rich plasma (PRP), venous leg ulcer

Chronic venous leg ulcers (Fig. 1) are a significant diagnostic and therapeutic challenge. In most patients, venous ulcer develops secondarily to chronic venous insufficiency. The recurrent infection affects up to $72 \%$ of cases, and after skin graft the annual relapse rate can be as high as $48 \%$ (Morimoto et al., 2014). Chronic nature of venous ulcer, tissue damage, wound exudate and the decreased partial oxygen pressure promote colonisation and uncontrolled microbial growth. Whereas the colonisation itself does not significantly alter healing process, except for the cases of host immune response, the increase in bacterial count and synergistic interaction between the individual species may hinder ulcer healing, leading to further tissue damage (Bowler, 2002).

Antimicrobial treatment is difficult and often insufficient as antibiotics have poor penetration to the infection site (Alinovi et al., 1986). Therefore the treatment includes improvement of blood flow to the affected site and other types of experimental therapies (Howard et al., 2008; Rosales et al., 2010). One of the therapeutic alternatives in refractory venous leg ulcers is platelet rich plasma (PRP) successfully used in orthopaedics, rheumatology, maxillofacial surgery or aesthetic medicine. PRP is a rich source of autologous growth factors,

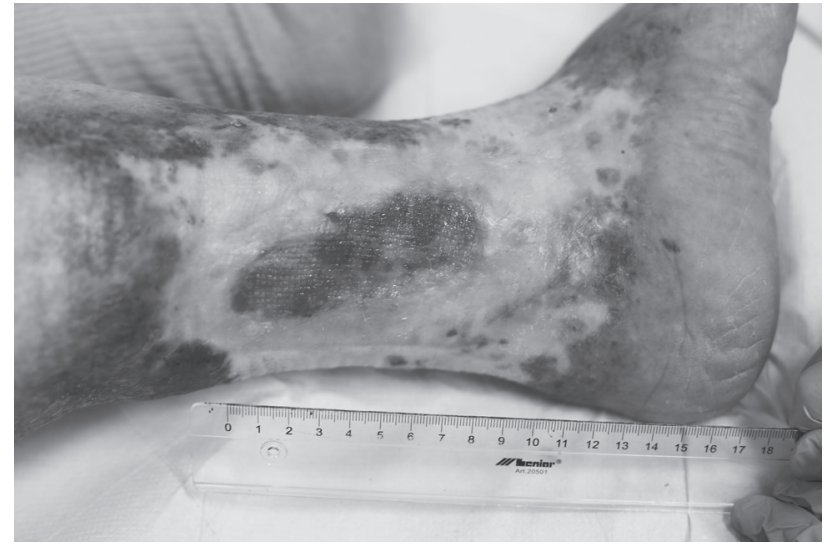

which participate in coagulation, immune response, angiogenesis and wound healing (Lubkowska et al., 2012). There are reports of improved ulcer healing after PRP therapy in comparison with the controls, as well as converse observations (Saad Setta et al., 2011; Martinez-Zapata et al., 2012). During our initial clinical attempts to treat venous leg ulcers with PRP, the more severe sings of microbial growth (increased inflammatory exudate, more intense smell of decomposition) were observed, hence the decision to address this issue.

\footnotetext{
* Corresponding author: W. Woźniak, First Department of General and Vascular Surgery, Medical University of Warsaw, Warsaw, Poland; e-mail: drww@onet.pl
} 
Therefore, we conducted a qualitative analysis of bacterial flora in venous leg ulcers during local treatment with platelet rich plasma.

Between 2012 and 2015, 34 patients with venous leg ulcers were treated at the First Department of General and Vascular Surgery, Second Faculty of Medicine with the English Division and the Physiotherapy Division, Medical University of Warsaw. The inclusion criteria for the treatment involved completed causal treatment, i.e. the absence of superficial and perforator vein reflux as well as the restored iliac vein patency. The exclusion criteria included leg ulcers secondary to atherosclerosis-related ischaemia, diabetes, rheumatoid arthritis, lymphatic obstruction or phlebitis. The essential eligibility criterion for local treatment with platelet rich plasma was the lack of progress of spontaneous ulcer healing despite completed causal treatment. The allowed time window was 6 months, i.e. if the ulcer has not resolved or has not presented signs of progressing reepithelialisation within this period, it was eligible for PRP therapy. The culture specimens from the ulcer surface were collected 7-14 days prior to treatment. The specimens were collected using collection and transport system swabs and tubes with Amies BO91 medium (ZTS Hagmed). The collected biological material was cultured on the growth media for aerobic bacteria, anaerobic bacteria and fungi using the streak plate method on Columbia Agar with 5\% Sheep Blood, Haemophilus agar, MacConkey agar, Chapman agar, Scheadler agar, Sabouraud Dextrose agar, as well as MRSA and Strep B chromogenic media. Antibiotic susceptibility of each bacterial and fungal isolate was determined using either automated method (Vitek 2 Compact, BioMerieux) or Kirby Bauer disk diffusion method. The MIC gradient strips (E-test, The bacterial colony count was not determined, but only roughly estimated based on the number of colonies grown after plating and noted for diagnostic purposes as: 1 - scanty, 2 - few, 3 - moderate, 4 - numerous.

Platelet rich plasma was prepared from $18-27 \mathrm{ml}$ of blood drawn with syringe from the superficial veins on the forearm. The blood was then transferred to sterile $9 \mathrm{ml}$ test tubes containing sodium citrate (Vacuette 9NC, Greiner Bio-One GmbH, Austria). After mixing, the tubes were left on the tube rack until the RBCs separated from the plasma. The process typically lasted for 3-4 hours. Afterwards, using the new syringe and venflon cannula (Vasofix Certo 16G 2" B. Braun Melsungen, Germany) the supernatant (plasma) was collected. The collected plasma was transferred to sterile $9 \mathrm{ml}$ test tubes (Vacuette Z Greiner Bio-One GmbH, Austria). The test tubes containing plasma were centrifuged for 15 minutes at $3000 \mathrm{rpm}$ (Low Speed Centrifuge 80-2C, Pioway Medical Lab Equipment Co., Ltd., China). After centrifuging, using the new syringe and venflon cannula (Vasofix Certo 16G 2" B. Braun Melsungen, Germany) the upper half content of the test tube was collected $(4.5 \mathrm{ml})$. The remaining part of plasma was mixed (Vortex-Genie 2 Scientific Industries INC, USA) for 30 seconds in order to obtain homogeneous platelet solution. $0.5 \mathrm{ml}$ of the obtained solution was collected for platelet count (Cell Dyn 1700 Abbott Diagnostics, USA). The assay was compared to the automated full blood count results. Platelet concentration ratio ranged from 2.2 to 4.1 . Next, $0.1 \mathrm{ml}$ of calcium chloride $(10 \%$ Calcium chloratum, WZF) was added to each test tube of prepared platelet rich plasma solution. The platelet preparation was administered to the ulcer margins as an intradermal injection, using the $1 \mathrm{ml}$ syringes with $30 \mathrm{G}$ needle $(0.3 \times-4 \mathrm{~mm})$. Additionally, mesotherapy was performed on ulcer surface and after that, the ulcer was covered with platelet rich plasma. After plasma agglutination on ulcer surface, the entire area was covered with a proper size sterile hydrogel wound dressing Aqua-Gel (Kikgel). Wound dressing was changed every 3 days so as to avoid removing the plasma from ulcer surface. Control cultures were collected 3 weeks after the PRP treatment. During that period no antibiotics - local or systemic - were administered. Treatment efficacy was assessed visually based on the ulcer size decrease and the re-epithelialisation.

The study group (34 subjects) consisted of 22 women (64.7\%) and 12 men (35.3\%) whose age ranged from 47 to 90 years. The mean age was 70.5 years (SD 9.8). The surface area of the ulcer ranged from 3 to $92 \mathrm{~cm}^{2}$ with the mean surface area of $31.94 \mathrm{~cm}^{2}$. Single ulcer was present in $25(73.5 \%)$ patients. Double ulcer was shown in $4(11.8 \%)$ patients, whereas 5 subjects (14.7\%) presented with multiple ulcers (3 or more). Symptom duration ranged between 11 and 192 months, with the mean duration of 55.8 months.

After PRP therapy, a significant healing improvement was shown in 21 subjects $(61.8 \%)$, as assessed by decrease in wound size, whereas in 13 remaining cases (38.2\%) there was no improvement.

Microbiological analysis of bacterial flora a in the wounds prior the treatment yielded 83 microbial isolates. The most commonly isolated bacterial species included (in the order of frequency): Pseudomonas aeruginosa, Staphylococcus aureus, Enterococcus faecalis and Bacteroides fragilis. Table I shows quantitative distribution of isolated bacterial and fungal species among patients. The majority of cultures from single swab from the patient $(73.5 \%)$ showed the presence of multiple species.

Gram-positive bacteria were isolated from over 30\% of patients, Gram-negative bacteria were isolated from $59 \%$ of patients, with anaerobic bacteria and fungi making up $9.6 \%$ and $1.2 \%$, respectively. The most commonly isolated Gram-positive bacteria were S. aureus, E. faeca- 
Table I

Quantitative distribution of microbial species before and after PRP therapy.

\begin{tabular}{|c|c|c|c|}
\hline \multirow[b]{2}{*}{ Type } & \multirow[b]{2}{*}{ Species } & \multicolumn{2}{|c|}{ No. of isolates } \\
\hline & & $\begin{array}{c}\text { before PRP } \\
\text { therapy }\end{array}$ & $\begin{array}{c}\text { after PRP } \\
\text { therapy }\end{array}$ \\
\hline \multirow[t]{9}{*}{ Gram-positive cocci } & Methicillin-susceptible Staphylococcus aureus (MSSA) & 10 & 16 \\
\hline & Enterococcus faecalis & 6 & 7 \\
\hline & Methicillin-resistant Staphylococcus aureus (MRSA) & 3 & 3 \\
\hline & Streptococcus group C & 2 & 2 \\
\hline & Streptococcus agalactiae (group B) & 2 & 3 \\
\hline & Streptococcus pyogenes (group A) & 1 & 0 \\
\hline & Streptococcus group G & 1 & 1 \\
\hline & Staphylococcus hominis & 0 & 1 \\
\hline & Staphylococcus lugdunensis & 0 & 1 \\
\hline \multirow[t]{11}{*}{ Gram-negative bacilli } & Serratia marcescens & 4 & 1 \\
\hline & Morganella morganii & 4 & 7 \\
\hline & Escherichia coli & 3 & 10 \\
\hline & Proteus mirabilis & 3 & 6 \\
\hline & Klebsiella oxytoca & 3 & 4 \\
\hline & Enterobacter cloacae & 3 & 5 \\
\hline & Pantoea agglomerans & 2 & 1 \\
\hline & Proteus vulgaris & 1 & 1 \\
\hline & Klebsiella pneumoniae & 1 & 1 \\
\hline & Aeromonas hydrophila & 0 & 2 \\
\hline & Hafnia alvei & 0 & 1 \\
\hline \multirow[t]{6}{*}{ Gram-negative non-fermenting bacilli } & Pseudomonas aeruginosa & 18 & 24 \\
\hline & Acinetobacter baumannii & 2 & 2 \\
\hline & Stenotrophomonas maltophila & 2 & 2 \\
\hline & Pseudomonas putida & 1 & 0 \\
\hline & Acinetobacter haemolyticus & 1 & 0 \\
\hline & Alcaligenes faecalis & 1 & 0 \\
\hline \multirow[t]{4}{*}{ Anaerobic bacteria } & Bacteroides fragilis & 6 & 6 \\
\hline & Bacteroides ovatus & 0 & 1 \\
\hline & Prevotella oralis & 2 & 0 \\
\hline & Prevotella denticola & 0 & 1 \\
\hline \multirow[t]{2}{*}{ Fungi } & Candida parapsilosis & 1 & 0 \\
\hline & Candida albicans & 0 & 1 \\
\hline
\end{tabular}

lis, and $\beta$-hemolytic streptococci (Table I). Analysis of antibiotic resistance revealed that among studied isolates, MRSA strains (Table I) and enterococci showing HLAR to gentamicin (3 isolates) were present. No vancomycin resistant enterococci (VRE) were isolated.

The most commonly isolated Gram-negative bacteria were non-fermenting bacilli, predominantly P. aeruginosa, Acinetobacter baumanii and Stenotrophomonas maltophilia. Enterobacteriaceae constituted large group of Gram negative bacteria isolated from ulcers. The most commonly isolated species were Serratia marcescens, Morganella morganii, Escherichia coli, Proteus mirabilis, Klebsiella oxytoca and Enterobacter cloacae.
No ESBL(+) Enterobacteriaceae strains were isolated. Two isolates of $P$. aeruginosa strains showed resistance to impenem and meropenem. B. fragilis was the most commonly isolated anaerobic bacterial species. Candida parapsilosis was isolated in one case.

After PRP therapy, a total of 110 bacterial isolates were obtained from samples collected after a single, local application of platelet rich plasma. Multiple isolates were cultured for 32 of 34 subjects.

The bacterial species isolated after the treatment were very similar to those isolated previously, however the frequency of MSSA, P. aeruginosa or E. coli isolation increased (Table I). There were no significant changes 
in antibiotic resistance profiles of isolated bacteria. Similarly to the initial cultures, no vancomycin resistant enterococci were isolated.

In this group of isolates, $5,1 \%$ strains were classified as ESBL positive and $8,3 \%$ of $P$. aeruginosa strains were resistant to impenem and meropenem. As previously, $B$. fragilis was the most commonly isolated anaerobic species. Candida albicans was isolated in a single case.

A comparison of the number of isolates in ulcer culture samples before and after PRP therapy showed an increase in the number of isolates containing multiple bacterial species. The maximum number of species in a single ulcer was five prior to PRP, it increased up to seven afterwards. The mean number of bacterial species isolated per subject before and after PRP was $2.44+/-0.22$ and $3.24+/-0.29$, respectively. Paired T-test comparing group of patients prior and after treatment shown that the increase in mean number of species is statistically significant $(\mathrm{P}=0.035)$.

A comparison of the most commonly isolated bacteria in culture samples before and after PRP therapy showed a marked increase in the isolation ratio of MSSA and E.coli, with changes in the ratio of other strains being less pronounced (Table I). Although quantitative analysis was not performed, visual estimation of the colony density on plates with plated bacteria after PRP therapy shows that the use of platelet rich plasma decreased the amount of bacteria in about half of the patients. However, based only on semi-quantitative inspection, we cannot speculate if the observed decrease in number of bacteria was statistically significant.

The influence of platelets on wound healing is well known. Chronic ulcers show decreased activity of growth factors, which delays tissue repair mechanisms. In 1974, Ross et al. found in their in vitro studies that thrombin activated platelets may constitute a rich source of growth factors. Thrombocytes release over 30 growth factors to the wound. These include three isomers of platelet derived growth factor - PDGF ( $\alpha \alpha$, $\beta \beta, \alpha \beta)$, vascular endothelial growth factor - VEGF, transforming growth factor $\beta$ - TGF- $\beta 1$, epithelial growth factor - EGF, insulin-like growth factor - IGF-1, angiopoietin 2 (Ang-2), interleukin $1 \beta$ (IL-1 $\beta$ ) and many others. Platelet rich plasma is also a source of proteins involved in cell adhesion, such as fibrin, fibronectin, vitronectin, osteocalcin or osteonectin (Everts et al., 2006). The growth factors are released as a result of degranulation of thrombocyte granules soon after the coagulation cascade is initiated and bind the membrane receptors of adjacent cells, thus activating the intracellular signalling pathway (Anitua et al., 2004; Eppley et al., 2004). The growth factors attract the undifferentiated cells within the wound, stimulate cell division, trigger the capillary growth and improve epithelial repair mechanisms. Additionally, they inhibit cytokine expres- sion, thus limiting the inflammatory response (Carter et al., 2011; Scimeca et al., 2010). Platelet rich plasma shows antibacterial properties, mainly due to PDGF, which activates macrophages and VEGF, which activates macrophages and monocytes. Furthermore, PRP contains leukocytes, which enhance its antibacterial properties. In vitro studies showed good efficacy of PRP against $S$. aureus (MSSA), which was comparable to the one of gentamycin and oxacillin (Cieślik-Bielecka et al., 2012). Li et al. (2013) observed a significant antibacterial activity of PRP against MRSA in experimentally induced osteitis in rats. Also Moojen et al. (2008) confirmed the efficacy of PRP against S. aureus. Bielecki et al. (2007) who studied the in vitro effect of platelet rich plasma provided a broad analysis of antibacterial properties of PRP. They assessed the antibacterial effect of PRP by measuring the inhibition zones on agar plates with individual microbial strains. PRP was shown to inhibit the growth of S. aureus, both MSSA and MRSA, as well as E. coli. The effect of PRP on K. pneumoniae, E. faecalis and P.aeruginosa was not confirmed. In the case of $P$. aeruginosa, PRP may even induce microbial growth (ref). Chronic ulcers are typically colonised by S. aureus and P. aeruginosa (Lim et al., 2006). As postulated by Gjodsbol et al. (2006), the presence of $P$. aeruginosa can particularly hinder ulcer healing and increase its surface area.

Our in vivo study shows that platelet rich plasma possibly inhibits growth of many bacterial species, including S. aureus and P. aeruginosa. However, based on a single study, it is difficult to determine whether this effect can be attributed to platelet rich plasma, microbial interactions, transfer of bacteria during wound dressing or other host factors. The use of PRP increased the number of microbial species in the healing wound. At the same time, the percentage of patients with culture-confirmed P. aeruginosa after PRP therapy has risen, which can be attributed to ulcer colonisation with nosocomial hospital strains. Nevertheless, these ulcerations tended to heal better despite the increased number of different microbial species. This may indicate colonisation rather than actual infection. Undoubtedly, the decreased colony count of most species was a beneficial effect likely to promote ulcer healing. This, however, requires further study.

In conclusion, local application of platelet rich plasma onto the surface of venous ulcers reduces the number of colonies, contributing to the increased variety of microbial flora at the same time.

\section{Literature}

Alinovi A., P. Bassissi and M. Pini. 1986. Systemic administration of antibiotics in the management of venous ulcers. A randomized clinical trial. J. Am. Acad. Dermatol. 15: 186-191. 
Anitua E., I. Andia, B. Ardanza, P. Nurden and A.T. Nurden. 2004 Autologous platelets as a source of proteins for healing and tissue regeneration. Thromb. Haemost. 91: 4-15.

Bielecki T.M., T.S. Gazdzik, J. Arendt, T. Szczepański, W. Król and T. Wielkoszyński. 2007. Antibacterial effect of autologous platelet gel enriched with growth factors and other active substances: an in vitro study. J. Bone Joint Surg. Br. 89: 417-420.

Bowler P.G. 2002. Wound pathophysiology, infection and therapeutic options. Ann. Med. 34: 419-427.

Carter M.J., C.P. Fylling and L.K. Parnell. 2011. Use of platelet rich plasma gel on wound healing: a systematic review and metaanalysis. Eplasty. 11: e38.

Cieślik-Bielecka A., D.M. Dohan Ehrenfest, A. Lubkowska and T. Bielecki. 2012. Microbicidal properties of leukocyte- and plateletrich plasma/fibrin (L-PRP/L-PRF): new perspectives. J. Biol. Regu. Homeost. Agents. 26: 43-52

Eppley B.L., J.E. Woodell and J. Higgins. 2004. Platelet quantification and growth factor analysis from platelet-rich plasma: implications for wound healing. Plast. Reconstr. Surg. 114: 1502-1508. Everts P.A., C. Brown Mahoney, J.J. Hoffmann, J.P. Schönberger, H.A. Box, A.van Zundert and J.T. Knape. 2006. Platelet-rich plasma preparation using three devices: implications for platelet activation and platelet growth factor release. Growth Factors. 24 165-171.

Gjødsbøl K, J.J. Christensen, T. Karlsmark, B. Jørgensen, B.M. Klein and K.A. Krogfelt. 2006. Multiple bacterial species reside in chronic wounds: a longitudinal study. Int Wound J. 3 225-231.

Howard D.P., A. Howard, A. Kothari, L. Wales , M. Guest and A.H. Davies. 2008. The role of superficial venous surgery in the management of venous ulcers: a systematic review.

Li G.Y., J.M. Yin, H. Ding, W.T. Jia and C.Q. Zhang. 2013. Efficacy of leukocyte- and platelet-rich plasma gel (L-PRP gel) in treating osteomyelitis in a rabbit model. J. Orthop. Res. 31: 949-956.
Lim T., B. Mwipatayi, R. Murray, K. Sieunarine, M. Abbas and D. Angel. 2006. Microbiological profile of chronic ulcers of the lower limb: a prospective observational cohort study. ANZ J. Surg. 76: 688-692.

Lubkowska A., Dołęgowska B. and G. Banfi. 2012. Growth factor content in PRP and their applicability in medicine. J. Biol. Regul. Homeost. Agents. 26: 3-22.

Martinez-Zapata M.J., A.J. Martí-Carvajal, I. Solà, J.A. Expósito, I. Bolíbar, L. Rodríguez and J. Garcia. 2012. Autologous plateletrich plasma for treating chronic wounds. http://onlinelibrary.wiley. com/doi/10.1002/14651858.CD006899.pub2/full, 2012.10.17. Moojen D.J., P.A. Everts, R.M. Schure, E.P. Overdevest, A. van Zundert, J.T. Knape, R.M. Castelein, L.B. Creemers and W.J. Dhert. 2008. Antimicrobial activity of platelet-leukocyte gel against Staphylococcus aureus. J. Orthop. Res. 26: 404-410.

Morimoto N., S. Takemoto, K. Kawai, R. Aya, T. Ishisaka and S. Suzuki. 2014. Immediate evaluation of neovascularization in a grafted bilayered artificial dermis using laser Doppler imaging. Ann. Plast. Surg. 72: 84-88.

Rosales A., G. Sandbaek and J.J. Jørgensen. 2010. Stenting for chronic post-thrombotic vena cava and iliofemoral venous occlusions: mid-term patency and clinical outcome. Eur. J. Vasc. Endovasc. Surg. 36: 458-465.

Ross R., J. Glomset, B. Kariyja and L. Harker. 1974. A plateletdependent serum factor that stimulates the proliferation of arte rial smooth muscle cells in vitro. Proc. Natl. Acad. Sci. USA 71: 1207-1210.

Saad Setta H., A. Elshahat, K. Elsherbiny, K. Massoud and I. Safe. 2011. Platelet-rich plasma versus platelet-poor plasma in the management of chronic diabetic foot ulcers: a comparative study. Int. Wound. J. 8: 307-312.

Scimeca C.L., M. Bharara, T.K. Fisher, H. Kimbriel and D.G. Armstrong. 2010. Novel use of platelet-rich plasma to augment curative diabetic foot surgery. J. Diabetes Sci. Technol. 4: 1121-1126. 
